\title{
Cost-effective Transceiver based on a RSOA and a VCSEL for Flexible uDWDM Networks
}

\author{
Jose A. Altabas, David Izquierdo, Jose A. Lazaro, Member, IEEE, and Ignacio Garces, Member, IEEE
}

\begin{abstract}
A cost-effective transceiver for a 1Gbps full-duplex uDWDM optical link is proposed for flexible metro-access and $5 G$ networks. The transceiver is based on a VCSEL, which is used as the LO for a heterodyne receiver and also feeds a phasemodulated RSOA transmitter. The modulation format used in the RSOA is a NRZ-DPSK for the uplink while the downlink is based on a Nyquist DPSK format. The central frequencies of the links are $2 \mathrm{GHz}$ separated and both links can be placed inside a $6.25 \mathrm{GHz}$ frequency slot. The sensitivity of this transceiver is $-43.5 \mathrm{dBm}$ over a $50 \mathrm{~km}$ fiber.
\end{abstract}

Index Terms - Coherent Receiver, Reflective Semiconductor Optical Amplifiers, Ultra Dense Wavelength Division Multiplexing, Vertical Cavity Surface Emitting Lasers

\section{INTRODUCTION}

$\mathrm{T}$ HE convergence of wireless and optical networks at the $5 \mathrm{G}$ scenario [1], combined with new streaming media and Internet of Things (IoT) services, are increasing the traffic of metropolitan and access networks. The evolution of these networks is converging to high capacity, all-optical merged-networks as the one shown in Fig. 1. In this context, flexible and coherent ultra-Dense Wavelength Division Multiplexing (uDWDM) metro-access networks are the most promising alternative to the current Time Division Multiplexing (TDM) optical networks due to its transparency and high spectral efficiency [2]. However, cost-effective devices have to be researched and developed in order to address the requirements of users and vendors [3].

This paper presents a cost-effective transceiver based on a continuous-emitting Vertical Cavity Surface Emitting Laser (VCSEL) [4], a phase-modulated Reflective Semiconductor Optical Amplifier (RSOA) [5] and a heterodyne coherent receiver. This transceiver allows a full-duplex $1 \mathrm{Gbps}$ channel

This work was supported in part by the Diputación General de Aragón under grant T25, the Spanish MINECO projects muCORE (TEC2013-46917C2-2-R) and SUNSET (TEC2014-59583-C2-1-R) co-funded by FEDER, Centro Universitario de la Defensa project SIRENA (CUD2013-05) and FPU grant from MECD to the first author (FPU 13/00620).

J.A. Altabas, D. Izquierdo, I. Garces are Grupo de Tecnologías Fotónicas (GTF), Aragon Institute of Engineering Research (I3A), Universidad de Zaragoza, Mariano Esquillor ed. I+D+i, Zaragoza, 50018, Spain (e-mail: jaltabas@unizar.es, d.izquierdo@unizar.es,ngarces@unizar.es).

D. Izquierdo is also with Centro Universitario de la Defensa (CUD), Academia General Militar, Carretera de Huesca s/n, Zaragoza, 50090, Spain.

J.A. Lazaro is with Grupo de Comunicaciones Ópticas (GCO), Universitat Politècnica de Catalunya, Jordi Girona 31, Barcelona, 08034, Spain (e-mail: jose.lazaro@tsc.upc.edu). inside a $6.25 \mathrm{GHz}$ frequency slot for a flexible uDWDM metro-access network with a sensitivity of $-43.5 \mathrm{dBm}$.

\section{SETUP}

The proposed transceiver is used in the Optical Network Unit (ONU) of the $1 \mathrm{Gbps}$ symmetrical link setup shown in Fig. 2. The key-component of the transceiver is the VCSEL which is used simultaneously, using a 50/50 coupler, as Local Oscillator (LO) in the reception stage and as feeder in the transmission stage. In the transmission stage, the VCSEL feeds an RSOA using another 50/50 coupler and an isolator to avoid the instability of the VCSEL cavity due to RSOA backscattering. This VCSEL has $20 \mathrm{MHz}$ of linewidth and the wavelength stability is $\pm 0.15 \mathrm{GHz}$ with a temperature stability of $\pm 0.01^{\circ} \mathrm{C}$. The RSOA, which is optical and electrically saturated with the VCSEL $\left(\mathrm{P}_{\mathrm{S}}=-5.5 \mathrm{dBm}\right)$ and with the $125 \mathrm{~mA}$ current bias, is modulated with 1 Gbps Non Return to Zero Differential Binary Phase Shift Keying (NRZ-DPSK) with $54 \mathrm{~mA}_{\mathrm{p}-\mathrm{p}}$. The ONU transmitted power $\left(\mathrm{P}_{\mathrm{TX}}\right)$ is $-3 \mathrm{dBm}$. The reception stage, that uses the VCSEL as $\mathrm{LO}\left(\mathrm{P}_{\mathrm{LO}}=-5.5 \mathrm{dBm}\right)$, is based on a single-photodiode heterodyne receiver [6] and can be easily upgraded to an independent polarization receiver [7].

The Optical Line Terminal (OLT) transceiver of this experimental setup is based on an external cavity Tunable Laser Source (TLS), located $2 \mathrm{GHz}$ shifted from the central wavelength of the ONU ( $f_{\mathrm{OLT}}=\mathrm{f}_{\mathrm{ONU}}-2 \mathrm{GHz}$ ), which is used as feeder for a Mach-Zehnder Modulator (MZM) at the transmission stage and as $\mathrm{LO}$ at the receiver stage. The MZM is set at the null point and modulated with a $1 \mathrm{Gbps}$ NyquistDPSK format with 12-symbols filter length and zero roll-off factor. The OLT transmits the same power than the ONU, $\mathrm{P}_{\mathrm{TX}}=-3 \mathrm{dBm}$. The OLT receiver is the same configuration that is used at the ONU but with different LO source (TLS) and power $\left(\mathrm{P}_{\mathrm{LO}}=0 \mathrm{dBm}\right)$. In a real implementation, the OLT could be implemented using as much transceivers as users or share

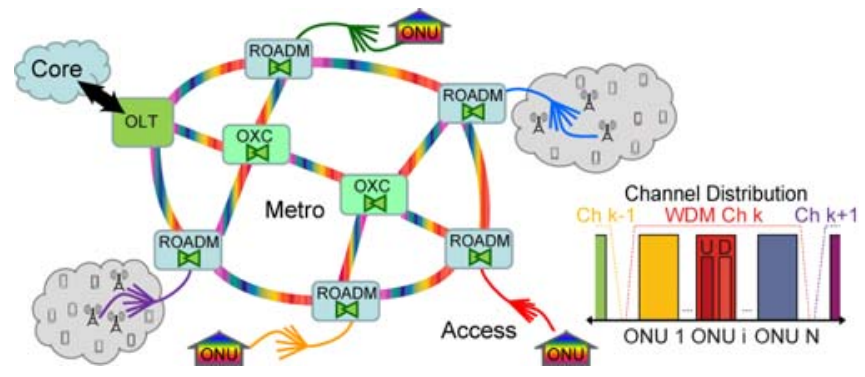

Fig. 1. All-optical 5G Metro-Access Network scenario. Inlet: proposed flexible uDWDM full-duplex channel distribution. 

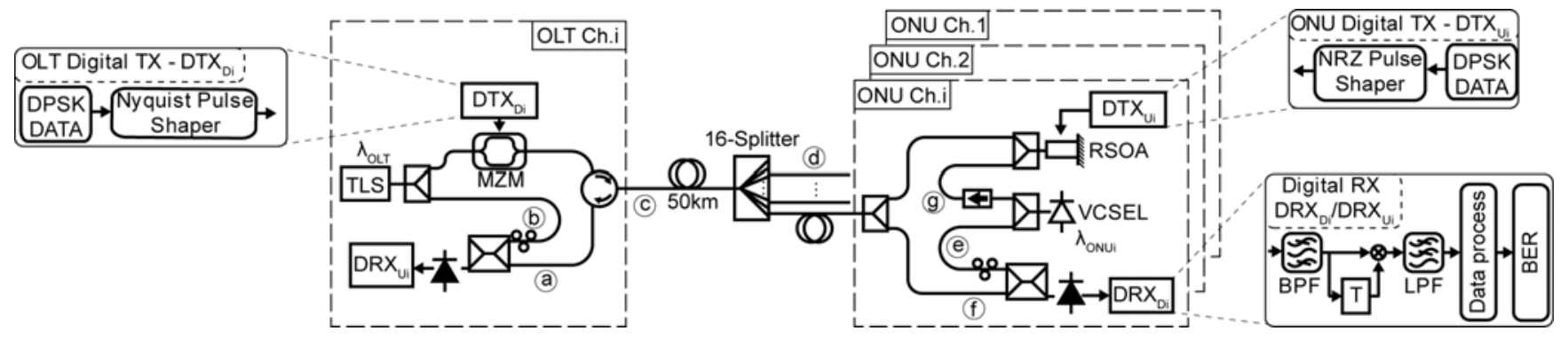

Fig. 2. Experimental setup for the evaluation of the optical link. $P_{\mathrm{Rx}}$ at (a) and (e) points, $P_{\mathrm{Lo}}$ at the (b) and (f) point, $P_{\mathrm{Tx}}$ at (c) and (d) points and Ps at (g) point. the OLT unit between several users and so reduce the cost [2, 8].

The transmitted signals for both transceivers are generated using a 12GS/s Arbitrary Waveform Generator (AWG) while the received signals are digitalized with a 40GS/s Digital Signal Oscilloscope (DSO) with $2.5 \mathrm{GHz}$ electrical bandwidth.

The optical channel is based on a $50 \mathrm{Km}$ Standard Single Mode Fiber (SSMF) and a 1:16 distribution splitter.

\section{RESUltS}

The sensitivity, defined as the minimum received power to ensure a BER of $2.2 \cdot 10^{-3}$ without FEC and $10^{-12}$ with a $7 \%$ overhead FEC [9], has been evaluated for both links in three different scenarios. The uplink sensitivity for back-to-back (BTB) transmission is $-48 \mathrm{dBm}$ while the power penalty due to a $50 \mathrm{Km}$ SSMF transmission is only $2 \mathrm{~dB}$, as shown in Fig. 3. The downlink sensitivity, shown in Fig. 4, is $-49 \mathrm{dBm}$ for BTB transmission and the power penalty due to fiber transmission is $1.5 \mathrm{~dB}$. The bidirectional connection, when both links are transmitting simultaneously, increases the power penalties by $1 \mathrm{~dB}(4 \mathrm{~dB})$ for the uplink (downlink). Consequently, the power budget in this one user scenario is $42 \mathrm{~dB}(40.5 \mathrm{~dB})$ for the uplink (downlink), so distances upper $100 \mathrm{Km}$ will be reached [6]. Equal power budget can be achieved by $\mathrm{P}_{\mathrm{TX}} / \mathrm{P}_{\mathrm{LO}}$ adjustment. A different OLT configuration, including additional users, could reduce the power budget.

The optical spectrum of the 1 Gbps symmetrical link, shown in Fig. 5, was obtained with a BOSA High Resolution Optical Complex Spectrum Analyzer (HROCSA) from Aragon Photonics Labs. The central wavelength of both links are shifted $2 \mathrm{GHz}$ away from each other and the wavelength used

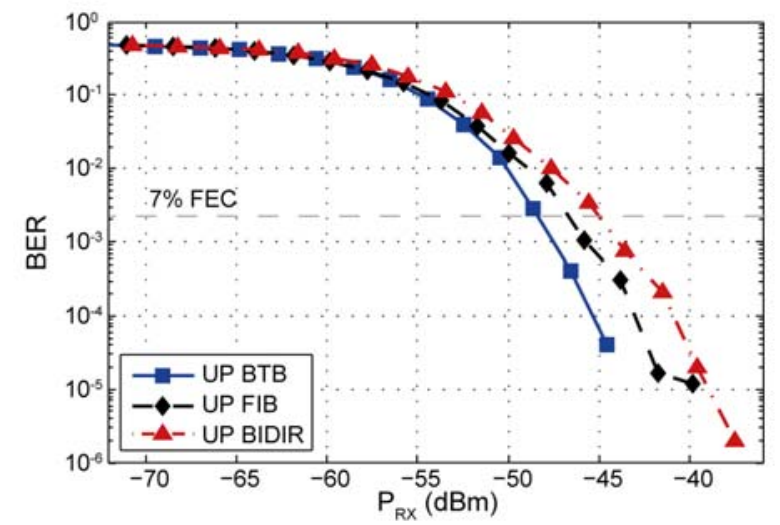

Fig. 3. BER vs received power for uplink for the BTB (UP BTB), fiber (UP FIB) and bidirectional connection (UP BIDIR).

for transmission is also used as LO for reception. The inlet of Fig. 5 shows the VCSEL spectrum used as light source in the proposed transceiver.

Fig. 5 can be used also to explain the asymmetrical power penalty in the bidirectional scenario. The backscattering of the downlink has a narrow bandwidth due to the Nyquist-DPSK modulation over one of the secondary lobes of the uplink spectrum and it can be easily electrically filtered with a small penalty in the OLT receiver. In contrast, the uplink (NRZ-DPSK) has a broad bandwidth and the backscattering generated by the secondary lobe introduces noise over all the downlink spectra, and it cannot be electrically removed. Thus, uplink has a lower power penalty than downlink in the full bidirectional channel scenario.

The uplink signal is generated with NRZ-DSPK modulated RSOA working as phase modulator. Therefore, transitions between symbols lie ideally on a constant amplitude circle at the IQ diagram. This transition between symbols implies a continuous phase variation, as can be seen at the optical phase eye diagram in Fig 6.a, obtained from the HROCSA. It can also be seen at the demodulated electrical eye diagram in Fig. 6.c, obtained from the implemented heterodyne receiver (Fig. 2). This eye diagram also shows a spread of the symbol amplitude caused by a residual amplitude modulation at RSOA. The optical IQ diagram from the HROCSA, shown at Fig. 6.e, confirms both phenomena. The continuous phase modulation of the RSOA is confirmed as the symbols transitions do not cross the IQ diagram origin, while the residual amplitude modulation generates transitions and symbols not laying on the amplitude constant circle.

The downlink signal is a Nyquist-DPSK over a MZM. In

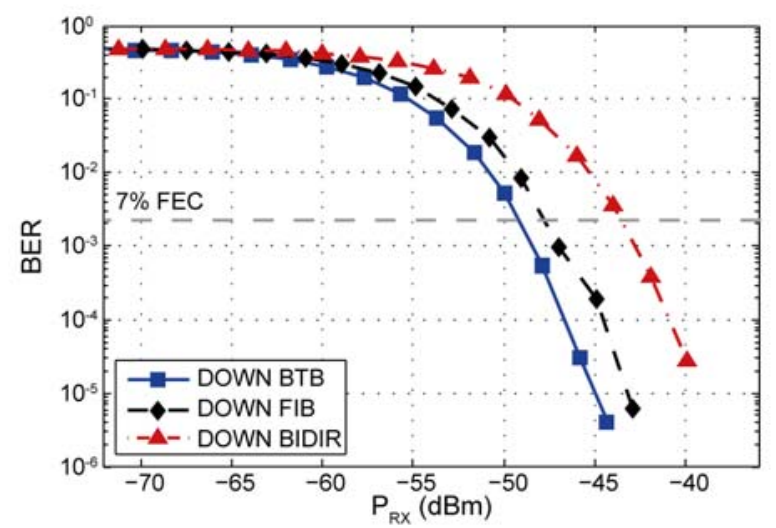

Fig. 4. BER vs. received power for downlink for the BTB (DOWN BTB), fiber (DOWN FIB) and bidirectional connection (DOWN BIDIR). 


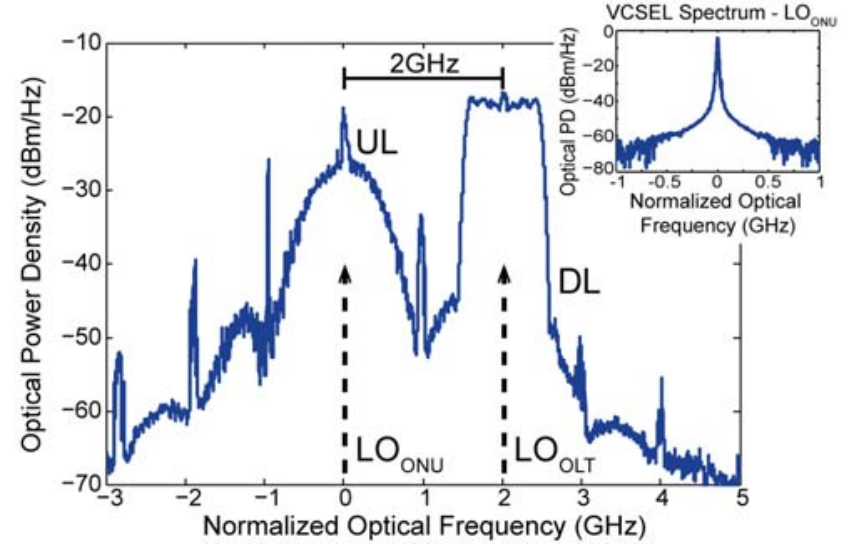

Fig. 5. Optical spectrum for both links of a single channel and the LO position of each link. The central frequency corresponds to $1523.58 \mathrm{~nm}$. Inlet: VCSEL Spectrum.

this case, the transitions between symbols cross the IQ diagram origin, Fig 6.f. The use of Nyquist pulse shaping introduces Inter Symbolic Interference (ISI) along the symbol period with the exception of the symbol center. Therefore, a noticeable temporal jitter at the optical phase transitions is measured Fig. 6.b. The demodulated eye diagram (Fig. 6.d), confirms this temporal jitter and shows that the signal maximum is not located in the optimal sampling point due to amplitude overshoots caused by the ISI. The optical IQ diagram (Fig. 6.f) confirms the transitions between symbols crossing the IQ diagram origin and the amplitude overshoots.

The interference between two adjacent channels has been analyzed when OLT is, simultaneously, receiving the uplink from $\mathrm{ONU}_{\mathrm{i}}$ while transmitting the downlink to an adjacent $\mathrm{ONU}_{\mathrm{i} \pm 1}$. In this scenario, the downlink backscattering of the adjacent channel will deteriorate the uplink BER depending on

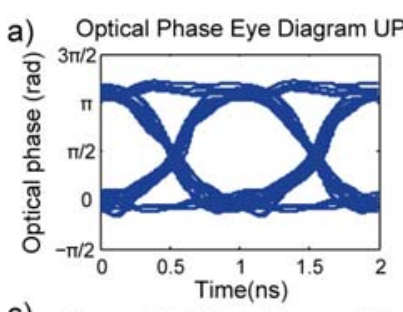

c) Demodulated Eye Diagram UP

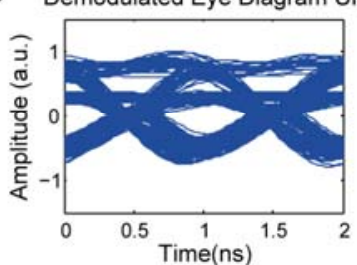

e)

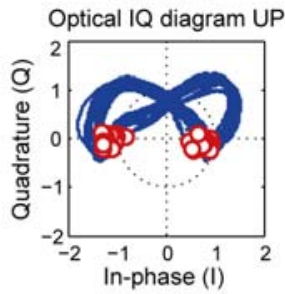

b) Optical Phase Eye Diagram DOWN

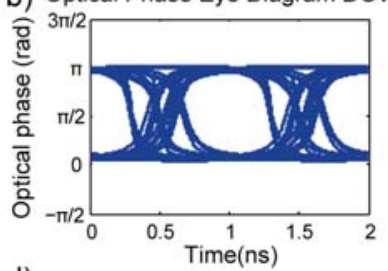

d) Demodulated Eye Diagram DOWN

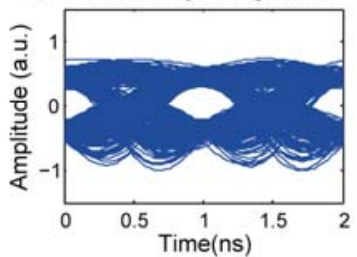

f)

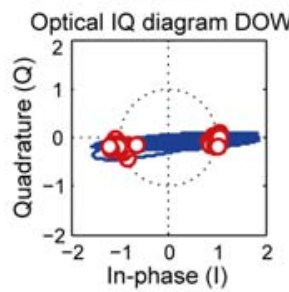

Fig. 6. Optical phase eye diagram from HROCSA ( $a$ and $b$ ), demodulated eye diagrams for $\mathrm{P}_{\mathrm{RX}}=-36 \mathrm{dBm}$ ( $\mathrm{c}$ and $\mathrm{d}$ ) and optical IQ diagram from HROCSA (e and f) for uplink (a, c and e) and downlink (b, d and f).

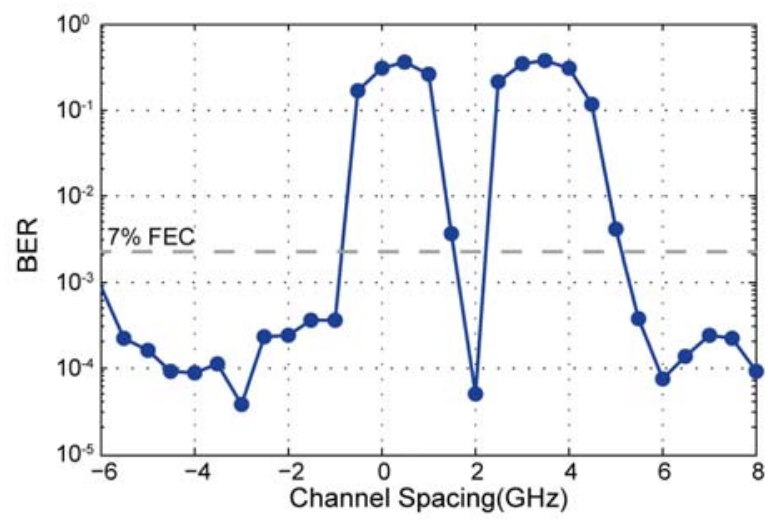

Fig. 7. Uplink BER at OLT while the OLT is transmitting the adjacent channel downlink.

the frequency distance of the adjacent channel. This is the only case studied because in our proposed channel distribution, the uplink is always flanked with two downlinks (the one of its channel and the one of the adjacent channel) and the interference of an adjacent uplink on the downlink is not relevant due the reduction of the uplink backscattering by the 1:16 distribution splitter [6]. The BER overpass the 7\% FEC limit for adjacent channel located in the range from $-1 \mathrm{GHz}$ to $1.5 \mathrm{GHz}$ and $2.5 \mathrm{GHz}$ to $5.5 \mathrm{GHz}$, as is shown Fig. 7. Therefore, frequency slots of $6.25 \mathrm{GHz}$ with null band guard [6] could be used for $1 \mathrm{Gbps}$ full-duplex link per user.

\section{CONCLUSION}

A full-duplex 1Gbps cost-effective transceiver has been evaluated for its integration in an uDWDM link for flexible 5G metro-access networks. This transceiver is based on a single continuous-emitting VCSEL, a phase-modulated RSOA and a heterodyne receiver. In the full-duplex scenario, the sensitivity is $-43.5 \mathrm{dBm}$, so ranges of more than $100 \mathrm{~km}$ can be reached. The uplink and the downlink are placed $2 \mathrm{GHz}$ away and the channels can be placed inside $6.25 \mathrm{GHz}$ frequency slots and grid.

\section{REFERENCES}

[1] M. Fiorani, et al., "Challenges for 5G transport networks", in Proc. ANTS, New Delhi. India, Dec. 2014, pp. 1-6.

[2] H. Rohde, et al., "Coherent Ultra Dense WDM technology for Next Generation Optical Metro and Access Networks", J. Lightw. Technol., vol. 32, no. 10, pp. 2041-2052, 2014

[3] M. Presi, et al., "All DFB-Based Coherent UDWDM PON With $6.25 \mathrm{GHz}$ Spacing and a $>40 \mathrm{~dB}$ Power Budget“, IEEE Photonics Technol. Lett., vol.26, no.2, 2014

[4] J.B. Jensen, et al., "VCSEL Based Coherent PONs", J. Lightw. Technol., vol. 32, no. 8, pp. 1423-1433, 2014

[5] H. K. Shim, H. Mu, U. H. Hong and Y. C. Chung, "A Practical 10-Gb/s Ultra-Dense WDM PON”, in Proc. OECC/ACOFT, Melbourne, Australia, Jul. 2014, pp. 289-290.

[6] Jose A. Altabas, et al., "1Gbps full-duplex links for ultra-dense-WDM $6.25 \mathrm{GHz}$ frequency slots in optical metro-access networks" Opt. Express, vol. 24, no.1, 555-565, 2016

[7] B. Glance, "Polarization independent coherent optical receiver", J. Lightw. Technol., vol. 5, no. 2, pp. 274-276, 1987.

[8] C. Kottke, et al., "Coherent UDWDM PON with joint subcarrier reception at OLT”, Opt. Express, vol.22, no. 14, pp.16876-16888, 2014.

[9] ITU-T Recommendation, G.975.1 (2004). 